MATEC Web of Conferences 33, 07001 (2015)

DOI: $10.1051 /$ matec conf/ 20153307001

(C) Owned by the authors, published by EDP Sciences, 2015

\title{
A novel approach to study the effect of heating temperature on prior- austenite microstructure of a Japanese sword
}

\author{
Anh Hoang Pham ${ }^{1, a}$, Takuya Ohba ${ }^{2}$, Shigekazu Morito ${ }^{2}$ and Taisuke Hayashi ${ }^{3}$ \\ ${ }^{1}$ Center for the Promotion of Research Project, Shimane University, Shimane 690-8504, Japan \\ ${ }^{2}$ Department of Materials Science, Shimane University, Shimane 690-8504, Japan \\ ${ }^{3}$ Department of Material Analysis, Shimane University, Shimane 690-8504, Japan
}

\begin{abstract}
There is uncertainty about heating temperature of final heat treatment and its effect on microstructure of Japanese sword, since the temperature is controlled entirely by experiences and skills of the swordsmith. This study proposed a novel approach to clarify how heating temperature affects prior-austenite microstructure of a Japanese sword. A fragment of the sword was normalized at $800^{\circ} \mathrm{C}$ to recover its initial microstructure. Specimens were cut and quenched, after being reheated at temperatures from 740 to $860^{\circ} \mathrm{C}$ with increment step of $20^{\circ} \mathrm{C}$. Their prior-austenite microstructure was reconstructed from martensite by using our newly developed automatic reconstruction method. In normalized microstructure of the sword, coarse pearlite islands of 0.70-0.76 mass\% C steel were found within a fine ferrite-pearlite matrix of 0.52-0.56 mass $\% \mathrm{C}$ steel. The prior-austenite grains of the medium $\mathrm{C}$ matrix remained fine $(11.6-15.1 \mu \mathrm{m})$ over the temperature range, while abnormal coarse grains $(73.9-123.7 \mu \mathrm{m})$ developed from high $\mathrm{C}$ islands in the specimens reheated above $780^{\circ} \mathrm{C}$. The prior-austenite microstructure of the original sword was closely similar to that of the specimen reheated at $760^{\circ} \mathrm{C}$. It was able to estimate the heating temperature of the original sword to be approximately $760 \pm 10^{\circ} \mathrm{C}$.
\end{abstract}

\section{Introduction}

Japanese sword is one of the most famous metal crafts of Japan. The sword is made from tamahagane, which is high quality steel made in the Japanese tradition [1-3]. The sword usually has a duplex construction (see Fig. 1), with the skin steel of high $\mathrm{C}$ content (0.6-0.7 mass\%), which responds to high strength of the body and hardness of the cutting edge [2-3]. The core is made of steel with low $\mathrm{C}$ content (0.2-0.3 mass \%) to improve the toughness [2-3]. To harden the cutting edge selectively, the sword is covered with a clay coating, which is thin at the sharp edge but thick at the main body. This special clay coating is used to control the cooling rate during final heat treatment of the sword [2].

During this step, the sword is heated to appropriate temperature followed by a fast quench in water. The edge with thin layer of coating is cooled rapidly and hardened by martensitic transformation, while the body with lower cooling rate transforms into a mixture of pearlite and ferrite with lower hardness but higher toughness [2-3]. The heat treatment is conducted in dim light, so the swordsmith can see well the color of the hot blade in order to judge its temperature. The heating temperature, which is said to be around $800^{\circ} \mathrm{C}$, is decided by the swordsmith experience [3].

From scientific point of view, this final heat treatment is one of the most important moments, when the sword

\footnotetext{
${ }^{\mathrm{a}}$ Corresponding author: anhpham@riko.shimane-u.ac.jp
}

obtains its final microstructure and mechanical properties. Along with the cooling rate controlled by the clay coating, the heating temperature is one of the key parameter, which mainly affects the evolution of prioraustenite microstructure (PAM). The latter has been reported to have a significant contribution to the strength and toughness of lath martensite [4-6], which is the final microstructure at the cutting edge of the sword [7-8]. Despite of its importance, there has not been any scientific study on the heating temperature and its effect on microstructure of Japanese sword.

In the present study, we proposed a novel approach to study the effect of heating temperature on the PAM of a Japanese sword, by using our new reconstruction technique for characterization of PAM of the specimens heat treated at different temperatures.

\section{Experimental}

The object of this study is a Japanese sword, which was prepared in 1945 by traditional method of sword making [2-3]. The chemical composition, microstructure and mechanical properties of this sword have been investigated by Yaso et al. [8-9]. According to their reports, the microstructure near the sharp edge of the sword is mainly lath martensite with 0.71 mass $\%$ of C, measured by electron probe micro-analysis (EPMA). 
In the present study, a $35 \mathrm{~mm}$ in length piece of the sword was used for investigation (Fig.1). Initially, that piece of sword was normalized at $800^{\circ} \mathrm{C}$ for 10 minutes to restore its ferrite-pearlite microstructure, which would probably be the initial microstructure of the sword [3, $10]$, prior to its final heat treatment.

After normalizing, seven cross-sectional specimens of $2.5 \mathrm{~mm}$ thickness were cut from the piece of sword. They were reheated in a salt bath at temperature varied from 740 to $860^{\circ} \mathrm{C}$ with increment step of $20^{\circ} \mathrm{C}$, followed by water quench to produce martensite in the skin steel. To be distinguished from specimen of original sword, those specimens will be referred as reheated specimens. The lower limit $740^{\circ} \mathrm{C}$ of heating temperature was chosen, because the $\mathrm{Ae}_{3}$ critical temperature of steel at the sharp edge with reported 0.71 mass $\% \mathrm{C}$ was calculated to be approximately $734^{\circ} \mathrm{C}[11]$.

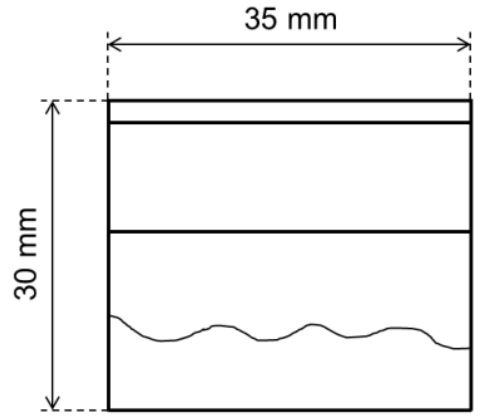

(Front view)

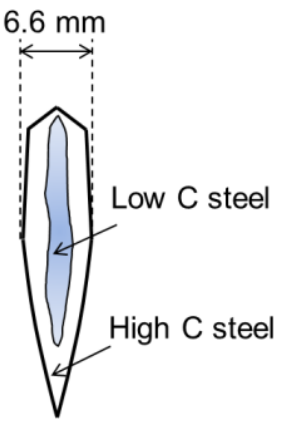

(Cross section)
Figure 1. Schematic illustration of one fragment of the sword used for investigation.

The microstructure of the specimens in normalized condition was observed by optical microscope. Orientations of martensite in seven reheated specimens and one specimen of the original sword were measured by electron backscatter diffraction (EBSD) technique. The EBSD experiments were carried out on $\mathrm{JEOL}^{\circledR}$ JSM7001FA field emission gun scanning electron microscope (SEM) equipped with $\mathrm{TSL}^{\circledR}$ EBSD data collection system.

In order to have large observation field, while maintaining sufficient resolution of martensite orientations, the magnification $\mathrm{x} 300$ was chosen. The EBSD scan was performed at $25 \mathrm{kV}$ of accelerating voltage and $0.25 \mu \mathrm{m}$ of scanning step. At this setting, the maximum scan area is $296 \times 865 \mu \mathrm{m}^{2}$ with 4.7 million of scan points. For each specimen, two or three scans were carried at different locations within a distance $4 \mathrm{~mm}$ from the sharp edge. This part in the original sword has been reported to be made of high $\mathrm{C}$ steel with full martensitic microstructure [8]. Hereafter, all the descriptions of microstructure and steel without any specific notation will be referred to this part of the sword.

The martensite orientations taken from EBSD data were used for reconstruction of austenite orientations, by using our automatic reconstruction software. Some details of our reconstruction method and software have been reported in [11-12]. After reconstruction, the PAM was characterized by calculation of prior-austenite grain
(PAG) size, fraction of primary twin boundary $(\Sigma 3)$ and fraction of secondary twin boundary ( $\Sigma 9)$. The calculation methods have been detailed in [11].

\section{Results and Discussions}

It is well known that the austenite grain in carbon and low-alloy steels grows with increase of heating temperature [13-14]. Moreover, the initial microstructure, which mainly determined by $\mathrm{C}$ content, also has strong effect on the development of PAM in $\mathrm{C}$ steel during heating $[11,15-17]$. Those matters will be investigated and discussed in this section.

\subsection{Initial microstructure and its impact}

After normalizing, the carbon inhomogeneity of the steel near the sharp edge of the sword was revealed through its optical micrograph (Fig. 2). The islands of coarse pearlite were observed within the matrix of very fine ferrite and pearlite. This specific microstructure is probably an inherent feature of the tamahagane steel, which is made through a number fold-forging sequences from a pile of raw steel pieces varied in $\mathrm{C}$ content [1-3]. However, the EPMA measurements on martensite of the original sword in the previous studies [8] have not detected this feature of the sword.

In order to estimate the $\mathrm{C}$ content of the fine- grained matrix and coarse pearlite islands, the area fraction of ferrite as grains of white contrast in the appropriate region on micrographs of two normalized specimens was calculated. The $\mathrm{C}$ content in mass $\%$ was derived from ferrite fraction using equation (1), which is based on lever rule, assuming similar mass density of ferrite and pearlite.

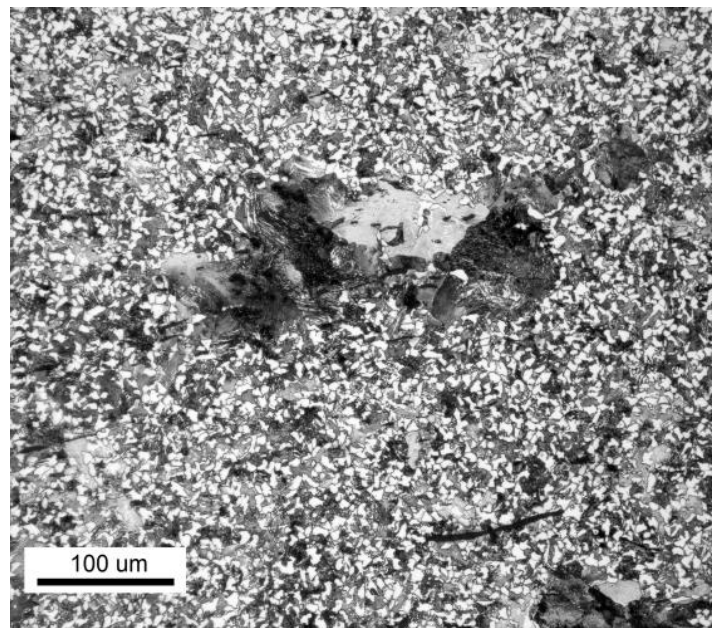

Figure 2. Optical micrograph taken near the sharp edge of normalized specimen, showing islands of coarse pearlite of high $\mathrm{C}$ steel (coarse grains with black and grey contrast) within the fine ferrite-pearlite mixture of the steel with lower $\mathrm{C}$ content.

$$
\% C=0.022+\left(1-a_{\mathrm{F}}\right)(0.761-0.022)
$$

In this equation, $a_{\mathrm{F}}$ is area fraction of ferrite measured on micrograph, while the constants 0.022 and 0.761 taken from [18], are the solubility of $\mathrm{C}$ in ferrite and austenite at eutectoid temperature $\left(727^{\circ} \mathrm{C}\right)$, respectively. 

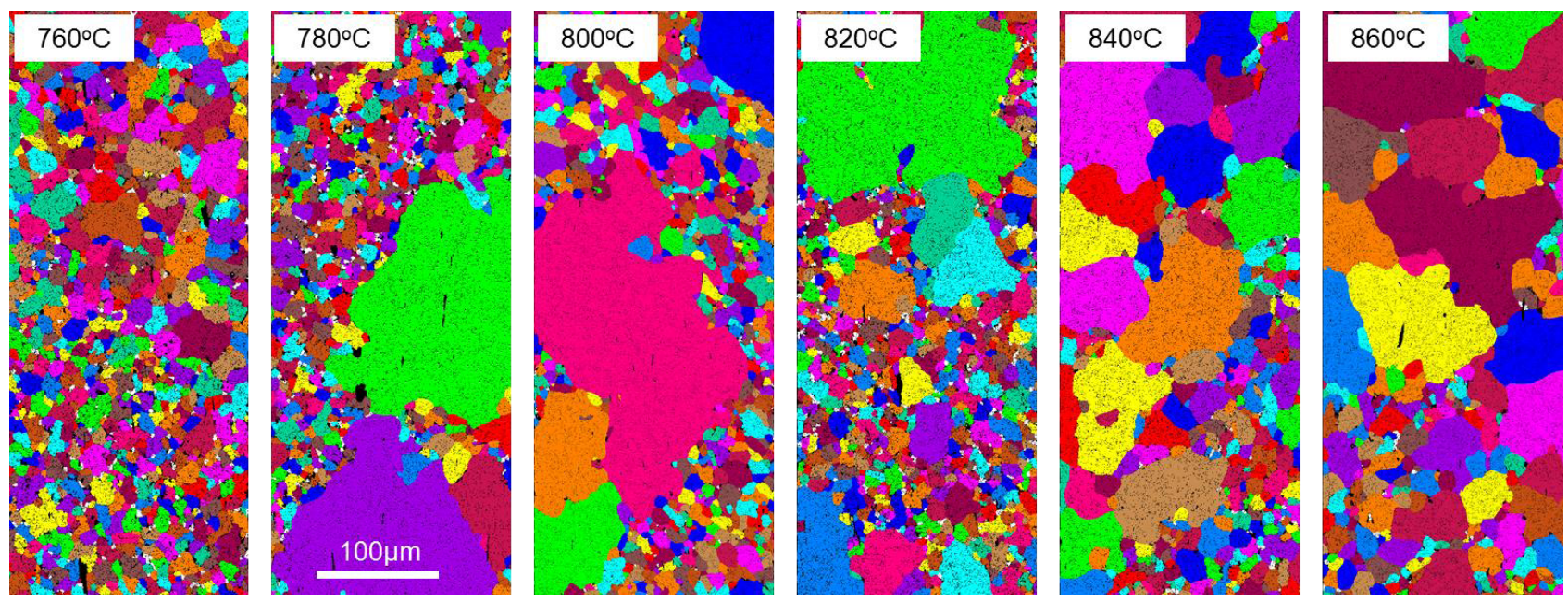

Figure 3. Typical austenite grain map of reheated specimens with increase of heating temperature, each grain was shaded by random color (the black spots are the defects of the steel or regions with undetectable austenite orientations, while the small white spots are the reconstructed austenite grains with diameter under a threshold of $3 \mu \mathrm{m}$ ).

It is found that the $\mathrm{C}$ content in fine-grained matrix is approximately $0.52-0.56$ mass $\%$. In coarse-grained islands, the value is $0.70-0.76$ mass $\%$. In some coarsegrained islands, the minor amount of cementite with light gray contrast might be omitted, and the maximum $\mathrm{C}$ content 0.76 mass $\%$ is taken from the case of full pearlitic microstructure. Because of lower $\mathrm{C}$ content in the matrix compared with the expected value of 0.71 mass \% C, for which the lower limit of heating temperature was determined, there is 0.11 fraction of retained ferrite in $740^{\circ} \mathrm{C}$ reheated specimen. However, no trace of ferrite is observed in other specimens reheated at $760^{\circ} \mathrm{C}$ or higher temperatures.

It is expected that the fine-grained matrix with medium $\mathrm{C}$ content will transform to fine austenite grains, while the coarse-grained pearlite with high $\mathrm{C}$ content will cause formation of coarse austenite grains at the given range of heating temperatures.

\subsection{Effect of heating temperature}

The effect of heating temperature on PAM of the sword is visible on the austenite grain map of reheated specimens (Fig. 3). A typical austenite grain map contains both finegrained and coarse-grained regions, which correspond to the fine ferrite-pearlite matrix and coarse pearlite islands observed in the initial microstructure. The reason has been discussed in [11] that during heating, in $\mathrm{C}$ steel with sufficient amount of ferrite, the PAG size is significant finer compared with the steels with low amount of ferrite or without ferrite in their initial microstructure.

Because of the abnormal grain distribution observed in Fig. 3, the PAG size was calculated separately for the fine and coarse-grained regions of medium and high $\mathrm{C}$ contents, respectively. The PAG size is plotted versus heating temperature in Fig. 4. It shows that the PAG size of medium $\mathrm{C}$ zones only changed slightly (from 11.6 to $15.1 \mu \mathrm{m})$ within the temperature range. On the other hand, the PAG size of high $\mathrm{C}$ zones increases sharply at $780^{\circ} \mathrm{C}$. After that a monotonous increase of grain size is not observed, but the PAG size even decreases slightly in a range from 123.7 to $73.9 \mu \mathrm{m}$. That behavior, which is different from the grain growth in ordinary steel [11, 13$15]$, is probably due to the variation of $\mathrm{C}$ content in the fine-grained matrix or coarse pearlite islands from one specimen to another. Nevertheless, when heating temperature is equal or higher $780^{\circ} \mathrm{C}$ the abnormally coarse austenite grains, which probably formed from coarse pearlite islands, are observed. Meanwhile, the austenite grains, which correspond to the fine ferritepearlite matrix, do not grow significantly over the range of heating temperatures.

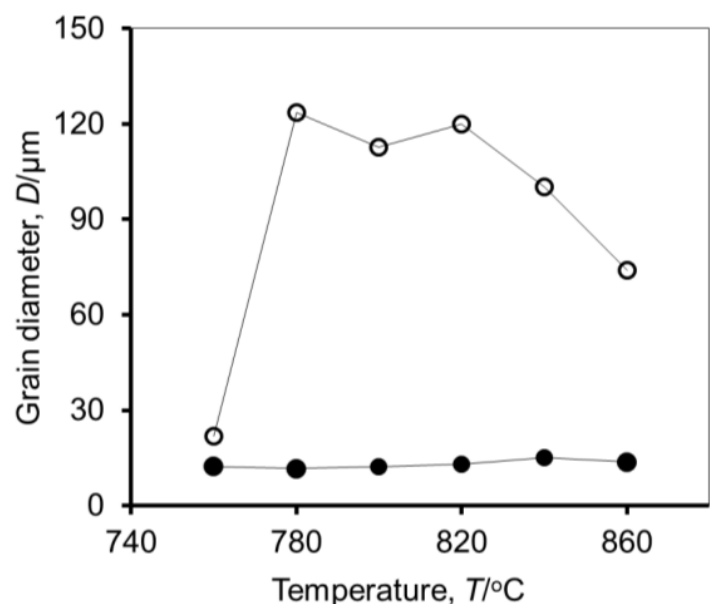

Figure 4. Variation of average austenite grain diameter in reheated specimens with temperature: the open and solid circles denote the values of coarse and fine-grained regions, respectively.

A careful check with four scans had been performed in different locations for the $760^{\circ} \mathrm{C}$ specimen, but the presence of abnormally coarse grains like in the other specimens was not found. The PAG size of coarsegrained zones in this specimen is only $21.8 \mu \mathrm{m}$, which is close to the grain size of fine-grained zones - $12.3 \mu \mathrm{m}$ (Fig. 4). We suppose that the normal grain distribution in that specimen is not due to the absence of coarse pearlite islands in its initial microstructure. It is probably that the grain growth of both medium and high $\mathrm{C}$ regions occurs with slow rate at that relatively low temperature. 
In a previous study [11], we have pointed out that the C content affects fraction of $\Sigma 3$ primary and $\Sigma 9$ secondary twin boundaries in PAM of $\mathrm{C}$ steels. In the present study, the fractions of those twin boundaries were also calculated from PAM of the specimens. The values were calculated separately for the same fine and coarsegrained zones, which have been used earlier for measurement of PAG size. The results are plotted with increase of heating temperature in Fig. 5.

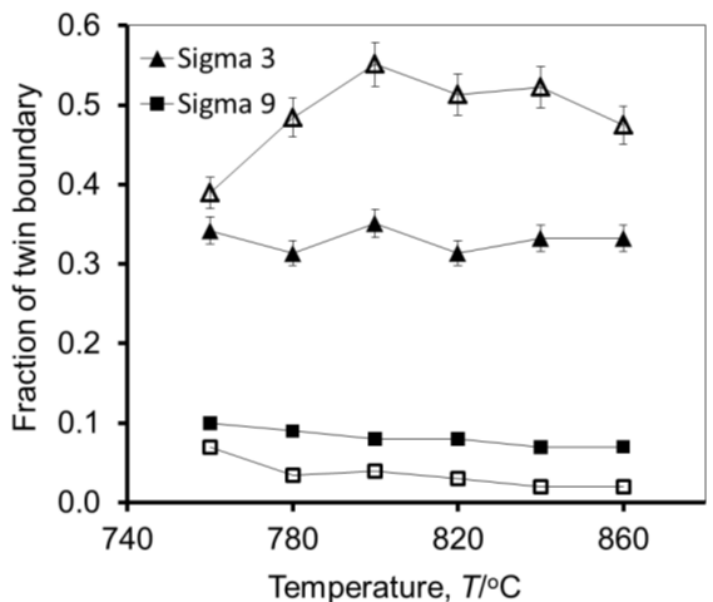

Figure 5. Variation of fractions of $\Sigma 3(\boldsymbol{\Delta})$ and $\Sigma 9(\mathbf{a})$ austenite twin boundaries with heating temperature: the open and solid symbols denote the values of coarse and fine-grained regions, respectively.

Except in the $760^{\circ} \mathrm{C}$ specimen, the fractions of $\Sigma 3$ and $\Sigma 9$ boundaries of fine and coarse-grained zones are well distinctive. For fine-grained austenite, the fraction of $\Sigma 3$ boundary is $0.29-0.35$, while the value is higher in coarse-grained austenite, $0.48-0.55$. On the contrary, the fraction of $\Sigma 9$ boundary is higher in fine-grained austenite $(0.07-0.10)$ compared with that in coarsegrained austenite $(0.02-0.04)$. This result, which is consistent with the previous observation in ordinary $\mathrm{C}$ steels with $0.38,0.61$ and 0.78 mass $\%$ of $C$ [11], supports our assumption of the different $\mathrm{C}$ contents in fine and coarse-grained austenite zones.

For $760^{\circ} \mathrm{C}$ specimen, the above tendency of variation in fractions of twin boundaries between fine and coarsegrained austenite is only slightly observed. The reason may be due to the similarity of PAG sizes, which causes more difficulty for separation of fine and coarse-grained austenite zones in that specimen. A considerable amount of fine austenite grains with medium $\mathrm{C}$ content may actually exist in the selected coarse-grained zone. Hence, the fraction of twin boundary obtained for coarse-grained austenite in $760^{\circ} \mathrm{C}$ specimen seems to be intermediate between the values for fine and coarse-grained zones in other specimens. For this reason, we will exclude the fractions of twin boundaries in coarse-grained zone of $760^{\circ} \mathrm{C}$ specimen from further discussions.

Compared with $\mathrm{C}$ content, heating temperature does not show a strong effect on the fractions of twin boundaries. The fraction of $\Sigma 9$ boundary only slightly decreases with increasing temperature for both fine and coarse-grained zones. Meanwhile, the fraction of $\Sigma 3$ boundary also varies in a narrow range (Fig.5).

\subsection{Heating temperature of the original sword}

Initially, this study was set up to discover the heating temperature of the original sword, by comparing its PAM with that of reheated specimens. However, the observed inhomogeneity of $\mathrm{C}$ in the sword make it difficult to find the heating temperature based solely on PAM. Nevertheless, a careful EBSD investigation with four scans on one specimen of the original sword has been carried.

As in $760^{\circ} \mathrm{C}$ specimen, the abnormally coarse grains were not found in all the observed PAM of the original sword. The average PAG size for fine and coarse-grained regions are 12.2 and $32.6 \mu \mathrm{m}$, respectively. The PAM of the two specimens is similar, although the size of coarse grains in the original sword is slightly higher than that in $760^{\circ} \mathrm{C}$ specimen. Moreover, the PAM of the sword is clearly different from that of other specimens, which were reheated from 780 to $860^{\circ} \mathrm{C}$.

All of these facts support our above assumption that the absence of abnormal coarse grains in $760^{\circ} \mathrm{C}$ specimen is not due to the nonexistence of high $\mathrm{C}$ islands in its initial microstructure, but it is because of the slower rate of austenite grain growth at relatively low temperature. It also allows concluding that the heating temperature of the original sword should be around $760^{\circ} \mathrm{C}$ because of the similarity in PAM observed in the two specimens. Taken a half of the temperature increment step as error of estimation, the heating temperature of the original sword used in this study could be approximately $760 \pm 10^{\circ} \mathrm{C}$.

\section{Conclusions}

The PAM of specimens from one Japanese sword, which were reheated at a range of temperatures, was characterized in order to reveal the effect of heating temperature. The followings results are obtained:

1) The inhomogeneous microstructure was observed in normalized specimens with coarse pearlite islands $(0.70-0.76$ mass $\% \mathrm{C})$ in a fine ferrite-pearlite matrix $(0.52-0.56$ mass $\% \mathrm{C})$.

2) The PAM of reheated specimens contains fine and coarse-grained zones, which correspond to the medium and high $\mathrm{C}$ locations in their initial microstructure.

3) The PAG size of fine-grained zones only increases slightly within the temperature range, while the PAG size of coarse-grained zones sharply increases at $780^{\circ} \mathrm{C}$.

4) The fractions of twin boundaries in PAM are clearly distinctive between fine and coarse-grained zones, but the values are virtually not affected by heating temperature.

5) The PAM of the original sword is closely similar to that of specimen reheated at $760^{\circ} \mathrm{C}$, so the heating temperature of the sword is determined approximately $760 \pm 10^{\circ} \mathrm{C}$. 


\section{Acknowledgement}

We are grateful to Wakoh museum of Japanese steel, for kindly providing the sample of the sword used in this study.

\section{References}

1. T. Kitamura, H. Katayama, Y. Murakawa, T. Okuno and T. Kishida, Ironmaking and Steelmaking 29, 276-280 (2002).

2. T. Inoue, Acta Mech. 214, 17-30 (2010).

3. L. Kapp, H. Kapp and Y. Yoshihara, The craft of the Japanese sword (Kodasha Int., Tokyo, 2007).

4. S. Morito, H. Yoshida, T. Maki and X. Huang, Mater. Sci. Eng. A 438-440, 237-240 (2006).

5. Y. Prawoto, N. Jasmawati and K. Sumeru, Mater. Sci. Technol. 28, 461-466 (2012).

6. G.A. Ostrovskii, V.I. Sarrak, K.Z. Shepelyakovskii and R.I. Entin, Met. Sci. Heat Treat. 6, 56-62 (1967).

7. A.K. Das, T. Ohba, S. Morito and M. Yaso, Mater. Sci. Forum 654-656, 138-141 (2010).
8. M. Yaso, T. Takaiwa, Y. Minagi, T. Kanaizumi, K. Kubota, T. Hayashi, S. Morito and T. Ohba, J. Alloys Compd. 577, S690-S694 (2013).

9. M. Yaso, Y. Minagi, T. Takaiwa, K. Kubota, T. Kanaizumi, T. Ohba, S. Morito and T. Hayashi, Mater. Sci. Forum 738-739, 222-227 (2013).

10. G. Takami, T. Ohba, S. Morito and A.K. Das, Mater. Sci. Forum 654-656, 134-137 (2010).

11. A.H. Pham, T. Ohba, S. Morito and T. Hayashi, Mater. Trans. (submitted).

12. A.H. Pham, T. Ohba, S. Morito and T. Hayashi: Mater. Trans. 54, 1396-1402 (2013).

13. S. Maropoulos, S. Karagiannis and N. Ridley, Mater. Sci. Eng. A 483-484, 735-739 (2008).

14. S.S. Zhang, M.Q. Li, Y.G. Liu, J. Luo and T.Q. Liu, Mater. Sci. Eng. A 528, 4967-4972 (2011).

15. V.I. Savran, Y.V. Leeuwen, D.N. Hanlon, C. Kwakernaak, W.G. Sloof and J. Sietsma, Metall. Mater. Trans. A 38, 946-955 (2007).

16. G.R. Speich and A. Szirmae, Trans. Metall. Soc. AIME. 245, 1063-1074 (1969).

17. G. Caballero, C. Capdevila and C.G. Andrés, ISIJ Int. 41, 1093-1102 (2001).

18. H. Okamoto, Ed., Phase diagrams of binary iron alloys (ASM international, Ohio, 1993). 
